\title{
Recommendations for lung cancer screening in Southern Africa
}

\author{
Coenraad F. N. Koegelenberg ${ }^{1}$, Shane Dorfman ${ }^{2}$, Ivan Schewitz ${ }^{3}$, Guy A. Richards ${ }^{4}$, Shaun Maasdorp ${ }^{5}$, \\ Clifford Smith ${ }^{6}$, Keertan Dheda ${ }^{7,8}$; on behalf of the South African Thoracic Society
}

${ }^{1}$ Division of Pulmonology, Department of Medicine, Stellenbosch University and Tygerberg Hospital, Cape Town, South Africa; ${ }^{2}$ Diagnostic Radiological Services (DRS) Inc., Alberton, South Africa; ${ }^{3}$ Department of Cardiothoracic Surgery, Pretoria University and Steve Biko Hospital, Pretoria, South Africa; ${ }^{4}$ Departments of Pulmonology and Critical Care, Faculty of Health Sciences, University of the Witwatersrand, Johannesburg, South Africa; ${ }^{5}$ Division of Pulmonology and Critical Care, Department of Medicine, University of the Free State and Universitas Academic Hospital, Bloemfontein, South Africa; ${ }^{6}$ Morningside Mediclinic, Sandton, South Africa; ${ }^{7}$ Centre for Lung Infection and Immunity, Division of Pulmonology, Department of Medicine and UCT Lung Institute \& South African MRC/UCT Centre for the Study of Antimicrobial Resistance, University of Cape Town, Cape Town, South Africa; ${ }^{8}$ Faculty of Infectious and Tropical Diseases, Department of Infection Biology, London School of Hygiene and Tropical Medicine, London, UK

Correspondence to: Coenraad F. N. Koegelenberg. Division of Pulmonology, Department of Medicine, Stellenbosch University \& Tygerberg Academic Hospital, PO Box 241, Cape Town, 8000, South Africa. Email: coeniefn@sun.ac.za.

\begin{abstract}
Lung cancer remains the leading cause of cancer-related deaths in southern Africa. Early trials of chest radiograph-based screening in males at high risk for lung cancer found no mortality benefit of a radiograph alone, or a radiograph plus sputum cytology screening strategy. Large prospective studies, including the National Lung Screening Trial, have shown an all-cause mortality benefit when lowdose computed tomography (LDCT) was used as a screening modality in patients that are at high risk of developing lung cancer. The South African Thoracic Society, based on these findings, and those from several international guidelines, recommend that annual LDCT should be offered to patients between 55-74 years of age who are current or former smokers (having quit within the preceding 15 years), with at least a 30-pack year smoking history and with no history of lung cancer. Patients should be in general good health, fit for surgery, and willing to undergo further investigations if deemed necessary. Given the high local prevalence of tuberculosis (TB) infection and post-TB lung disease, which can radiographically mimic lung cancer, a conservative threshold (nodule size $\geq 6 \mathrm{~mm}$ ) should be used to determine whether the baseline LDCT screen is positive (thus nodules $<6 \mathrm{~mm}$ require no action until the next annual screen). If a non-calcified, solid or partly solid nodule is $\geq 6 \mathrm{~mm}$, but $<10 \mathrm{~mm}$ with no malignant features (e.g., distinct spiculated margins), the LDCT should be repeated in 6 months. If a solid nodule or the largest component of a non-solid nodule is $\geq 10$ or $\geq 6 \mathrm{~mm}$ and enlarging or with additional malignant features present, definitive action to exclude lung cancer is warranted. Patients should be screened annually until 15 years have elapsed from date of smoking cessation, they turn 80 , become unfit for a curative operation or significant changes are observed.
\end{abstract}

Keywords: Lung cancer; screening; low dose computed tomography

Submitted Jul 09, 2019. Accepted for publication Aug 01, 2019.

doi: $10.21037 /$ jtd.2019.08.66

View this article at: http://dx.doi.org/10.21037/jtd.2019.08.66

\section{Introduction}

Lung cancer is the leading cause of cancer-related deaths in the world among men and woman (1). Worldwide more than 2 million new cases and almost 1.8 million deaths from lung cancer occurred in 2018 (1). In South Africa, lung cancer similarly ranks as the number 1 cause of cancer deaths (2) with the age standardised incidence rate (ASR) $3.95 / 100,000$ in females and 10.12/100,000 in males (3).

The most important risk factor for lung cancer remains tobacco smoking. It is estimated that $33.4 \%$ of males and $8.3 \%$ of females above the age of 15 are consumers of 
tobacco in South Africa (4). Other factors such as a family history, poor diet, chronic obstructive pulmonary disease (COPD), ionising radiation, human immunodeficiency virus (HIV) infection, occupational exposures and air pollution may also predispose to lung cancer (5).

The prognosis for non-small cell lung cancer is directly related to the stage at the time of diagnosis. Based on the current TNM classification, five-year survival using clinical staging ranges from $92 \%$ (stage IA) to zero (stage IVB) (6). In the USA, only $25 \%$ of patients with lung cancer are potentially amenable to cure on presentation (7). In South Africa, the outlook is even bleaker, as $<10 \%$ of patients have potentially curable disease at presentation (8). There is even evidence to suggest that having access to private medical care does not necessarily influence the tendency to present too late (9).

At face value, screening for lung cancer seems highly appropriate, given that smoking is the major identified risk factor (which allows the targeting of high-risk individuals) along with the high prevalence of lung cancer, the high associated morbidity and mortality, the protracted preclinical phase, and the clear evidence that therapy is more effective the earlier the diagnosis is made (10). For lethal diseases such as lung cancer, disease-specific outcomes and overall mortality are the most important outcomes when assessing the value of screening (10). In addition to the possibility of mortality reduction due to earlier disease detection, evidence suggests that participation in a randomised controlled trial (RCT) with low-dose computed tomography (LDCT) scan has a positive impact on smoking cessation (11). Finally, there may be other potential benefits including the detection of coronary artery disease (through coronary artery calcification scores) and identification of those with a higher risk of a cardiovascular event (12).

Lung cancer screening, on the other hand, may have certain disadvantages, particularly in resource-constrained settings (13). Diagnostic interventions come at a financial burden, some morbidity, and sometimes even mortality (13). "Overdiagnosis" bias is also a well-known phenomenon and early diagnosis may also not improve survival in those with significant comorbidities (14). Moreover, radiation exposure from LDCT may over time result in new lung and other cancers developing (15). That said, there is supporting evidence to suggest that the benefits outweigh the risks in that one lung cancer death may be avoided per 320 scanned (16). To put this in perspective, mammography saves one life per
1,339 patients scanned (16).

Southern Africa is faced with the "colliding epidemics" of smoking, HIV and TB, and despite robust international evidence to support lung cancer screening in appropriate individuals, no local data exist (17). It is within this context and background that the South African Thoracic Society (SATS) set out to propose local criteria for appropriate lung cancer screening.

\section{Chest radiograph and sputum cytology}

Lung cancer screening with chest radiography and/ or sputum cytology has been evaluated in several large prospective studies dating as far back as the 1960s, and no single trial has shown a mortality benefit (18). The Prostate, Lung, Colorectal, and Ovarian (PLCO) cancer screening trial was the largest randomised trial $(n=154,942$ participants) performed and it demonstrated that annual screening with a chest radiograph did not reduce lung cancer mortality (19).

\section{Low-dose chest computed tomography}

\section{General technical aspects and safety}

Modern multidetector LDCT imaging is designed to minimise radiation dose while still achieving high quality diagnostic images. Today's modern multidetector CT scans obtain adequate resolution while delivering a median effective dose of $1.0 \mathrm{mSv}$ for males and $1.4 \mathrm{mSv}$ for females, compared to $7 \mathrm{mSv}$ for a standard diagnostic chest CT and $0.1 \mathrm{mSv}$ for a chest radiograph (20). As a further reference, the annual average of the natural background radiation in the USA is $3 \mathrm{mSv}$, and is even higher in some parts of South Africa, e.g., Paarl (20,21).

LDCT are performed without contrast during a single maximal inspiratory breath-hold with a scanning time of less than 25 seconds (22). High-resolution images (1.0-2.5 mm interval) are reconstructed by means of a soft tissue or thinsection algorithm (20).

While $\mathrm{mSv}$ is an appropriate measurement for a population, CT dose Index (CTDI) volume is more appropriate when considering radiation exposure in an individual. The American college of Radiologists (ACR) guideline recommends a CTDI volume of $<3 \mathrm{mGy}$ for an average sized patient (23). This would be adjusted downwards for a smaller patient and upwards for a larger 
Table 1 Positive result definition of various screening (28)

\begin{tabular}{|c|c|c|}
\hline Program & Positive & Indeterminate \\
\hline NELSON & $\begin{array}{l}\text { A solid component of any non-calcified nodule } \geq 500 \mathrm{~mm}^{3}(>9.8 \mathrm{~mm} \text { in } \\
\text { diameter) }\end{array}$ & $\begin{array}{l}\text { Volume of the largest solid nodule or of the solid } \\
\text { component of a partially solid nodule: } 50-500 \mathrm{~mm}^{3} \\
\text { (4.6-9.8 } \mathrm{mm} \text { in diameter); or a nonsolid nodule } \geq 8 \mathrm{~mm}\end{array}$ \\
\hline ITALUNG & $\begin{array}{l}\text { At least one non-calcified solid nodules } \geq 5 \mathrm{~mm} \text { or a non-solid } \\
\text { nodule } \geq 10 \mathrm{~mm} \text { or the presence of a part-solid nodule }\end{array}$ & $\mathrm{N} / \mathrm{A}$ \\
\hline
\end{tabular}

NLST, National Lung Screening Trial; I-ELCAP, International Early Lung Cancer Action Project; NELSON, Nederlands-Leuvens Longkanker Screenings Onderzoek; ITALUNG, Italian Lung; N/A, not applicable.

patient (as appropriate) (23).

\section{General interpretation and reporting}

Appropriate window settings should be used to visualise all anatomical structures in the examination. Lung nodules should be described with respect to location, size, margins, density (solid, ground glass or part solid) and presence of calcification and/or fat (24). Furthermore, comparison with previous imaging, if available, is paramount in order to assess for interval change of nodules, and to evaluate if there are new nodules. Computerized decision support tools are useful adjuncts for nodule detection and volumetric assessment, and should be used when available $(25,26)$. In order to standardize CT reporting and management recommendations, to decrease ambiguity in interpretation, and to facilitate monitoring of outcomes, standard guidelines should be followed. The Lung-RADS ${ }^{\mathrm{TM}}$ classification system, created by the ACR provides such a guideline and SATS recommends the use of the current revised The Lung-RADS ${ }^{\mathrm{TM}}$ version 1.1 as the standard reporting guideline. The use of automated Lung-RADS ${ }^{\mathrm{TM}}$, where available, would not only assist the reporting radiologist to become more accurate and efficient in nodule detection and categorisation (25-27), but also access the benefits of the standardised interpretation, reporting and management recommendations provided by Lung-RADS ${ }^{\mathrm{TM}}$ itself.

\section{Evidence}

The National Lung Screening Trial (NLST) was a randomised trial comparing annual screening by LDCT with a chest radiograph at 33 centres in the USA for three years in more than 50,000 high-risk individuals (at least 30 pack years of smoking and aged 55-74 years) (Table 1) (16). The participants were randomly assigned to undergo annual screening with both modality for 3 years. Nodules or other suspicious findings were classified as positive results. In total, $27.3 \%$ of patients in the low-dose CT group and $9.2 \%$ in the radiography group had a positive screening result. Lung cancer was diagnosed in $1.1 \%$ in the lowdose CT group versus $0.7 \%$ in the radiography group. For those undergoing at least one screen, the number needed to screen with LDCT to prevent one lung cancer death was 320 and the NLST was terminated early after an interim analysis found a statistically significant benefit for LDCT scanning (16). At a median follow-up of 6.5 years, there were 645 cases of lung cancer per 100,000 person-years in the LDCT group, and 572 cases per 100,000 person-years in the chest radiograph group, resulting in an incidence rate ratio of 1.13 (95\% CI: 1.03-1.23). Lung cancers detected by screening were mostly stage I or II (70\% CT detected $v s$. $57 \%$ radiograph detected). Per 100,000 person years, there were 247 lung cancer deaths in the CT group and 309 in the radiograph group, yielding a relative mortality reduction of $20 \%$ (95\% CI: $3.8-26.7$ ) and an absolute reduction of 62 lung cancer deaths per 100,000 person years. Of significance was the $6.7 \%$ (95\% CI: $1.2-13.6)$ relative reduction in all-cause mortality in the LDCT group and an absolute reduction of 74 deaths per 100,000 person-years. Fewer stage IV cancers were observed in the LDCT group than the chest radiograph group with the second and third screening rounds, suggesting that diagnosis of earlier-stage cancers reduced the occurrence of later-stage lung cancers.

The Nederlands-Leuvens Longkanker Screenings Onderzoek (NELSON) trial was a RCT comparing LDCT 
screening at increasing intervals $(1,2$, and 2.5 years) with no screening in almost 16,000 current or former smokers in the Netherlands and Belgium (Table 1) (11,29-31). Unlike other screening studies, five-year lung cancer survivors, a group at very high risk of developing a new lung cancer, were eligible for enrolment. In a prespecified analysis, after a median of 8.16 years of follow-up, lung cancer was detected in $3 \%$ of the screened participants, with two thirds being stage 1 disease (31).

In the International Early Lung Cancer Action Project (I-ELCAP), screening resulted in a diagnosis of lung cancer in 484 participants. Of these, $412(85 \%)$ had clinical stage I lung cancer, and the estimated 10-year survival rate was $88 \%$ in this subgroup (95\% CI, 84-91). Among the 302 participants with clinical stage I cancer who underwent surgical resection within 1 month of diagnosis, the survival rate was $92 \%$ (95\% CI, 88-95). The eight participants with clinical stage I cancer who did not receive treatment died within 5 years of the diagnosis.

A retrospective interpretation of data from the I-ELCAP study cohort and NLST suggested that setting a more conservative threshold of $\geq 6 \mathrm{~mm}$ would decrease the falsepositive rate (resulting in fewer unnecessary procedures or follow-up studies) with minimal impact on the detection of cancers $(32,33)$. As such, compared with the NLST, the Lung-RADS criteria have a more conservative threshold for a positive baseline screen $(\geq 6 \mathrm{~mm})$ and require pre-existing nodules to demonstrate growth $(27,34)$.

\section{Lung cancer screening in Southern Africa}

\section{Patient selection and practical considerations}

Screening should only be performed in centres with requisite expertise in LDCT and lung cancer management, and only when the clinician and patient are committed to pursuing further investigations, including imaging and possible biopsy (and where appropriate, surgery or other modalities of cancer therapy) (35). Patients at risk need to be identified, and appropriately counselled. Lung cancer screening in southern Africa, as is the case globally, remains a multidisciplinary team effort with commitment from the funders, the community, the clinicians, the radiologists and ultimately the patient.

For individuals who opt to be screened after appropriate counselling, SATS recommends that annual screening with LDCT scanning only be available for those who meet all of the following criteria:
* Age 55-74 years;

* Current or former smokers (having quit within the preceding 15 years) with at least a 30 pack year history;

* No history of lung cancer;

* Are in general good health and fit for surgery.

Patients should be screened annually until 15 years have elapsed from date of smoking cessation, they turn 80, become unfit for a curative operation or significant changes (see below) are observed.

\section{Suggested LDCT protocols within a TB-endemic setting}

Protocols will be specific to each scanner as variability exists among scanners. There are, however, minimum equipment specifications required to achieve the recommended radiation targets; these include (23):

* Gantry rotation times of $\leq 0.5$ seconds;

* Slice thickness of $\leq 2.5 \mathrm{~mm}$ (preferably $\leq 1.0 \mathrm{~mm}$ );

* Detector rows of $\geq 16$;

* The CT scanner and/or the viewing platform should be capable of generating maximum intensity projection (MIP) and multiplanar reformation (MPR) images.

SATS recommends that the examination guidelines set by the American College of Radiology should be followed, including that (23):

* LDCT should be performed without contrast during a single maximal inspiratory breath-hold with a scanning time of less than 25 seconds (22);

* The field of view must include axial images from the lung apices to the costophrenic sulci;

* The examination should be acquired at $\leq 2.5 \mathrm{~mm}$ slice thickness (soft tissue may be viewed at $2.5 \mathrm{~mm}$ );

* Lung algorithms should be reconstructed at $\leq 1.0 \mathrm{~mm}$ slice thickness to allow for better characterization of small lung nodules;

* Use of MIP reconstruction to increase lung nodule detection and MPR to further characterize nodules, particularly along the pleural/fissural surfaces.

There is a high burden of TB in southern Africa with South Africa, according to the 2018 Global Report, having the highest incidence amongst the 30 high burden countries (36). Granulomas from previous TB infection (which may calcify) and those due to active disease frequently manifest as solitary or multiple nodules in any part of the lung (though the upper zones predominate). 


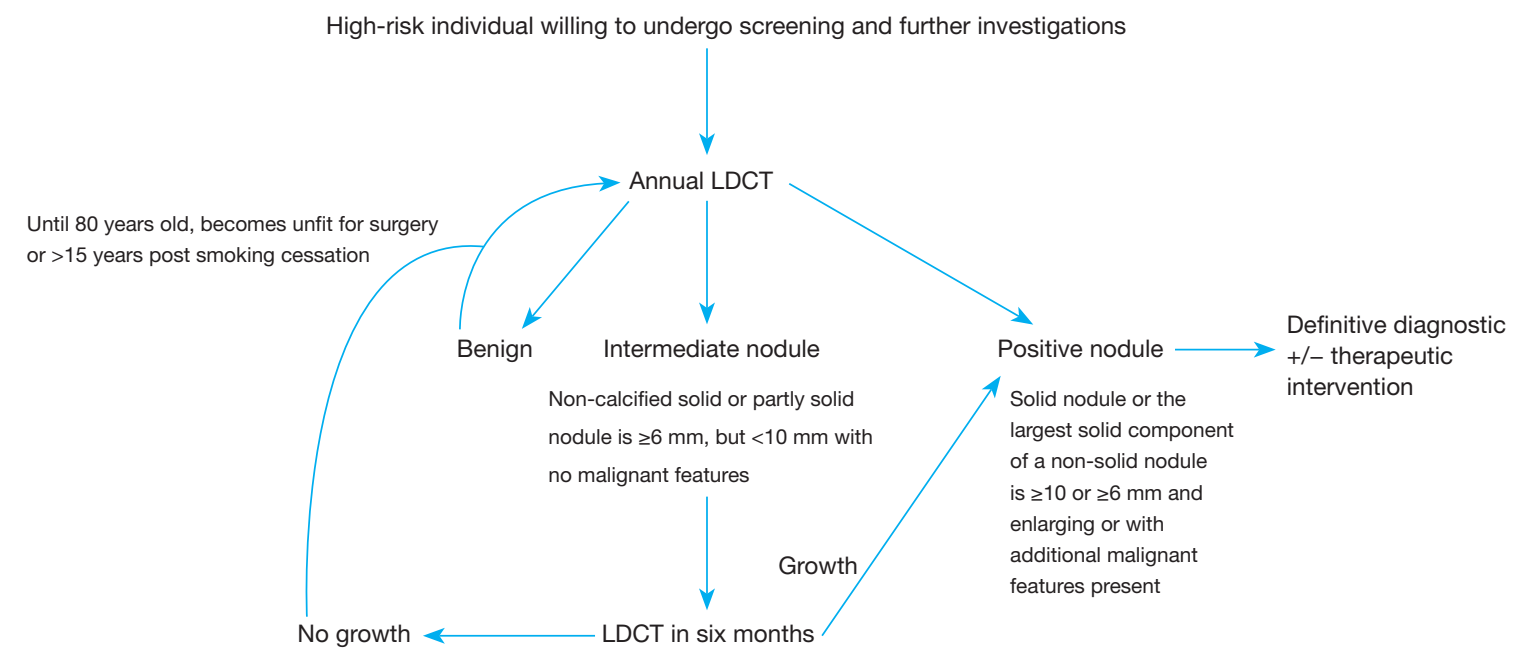

Figure 1 A suggested algorithm for lung cancer screening in southern Africa. High-risk individuals are those 55-74 years who are current or former smoker (having quit within the preceding 15 years) with at least a 30-pack year history, have no history of lung cancer and are in general good health and fit for surgery. LDCT, low-dose computed tomography.

Solitary pulmonary nodules may also be due to nontuberculous mycobacteria and should be considered even in TB endemic regions particularly where the incidence of HIV co-infection is high (37). The natural history of SPN due to TB is unclear but disease progression may be slow over the short term (less than 4 years) (38). Given these considerations, and the lack of local data on the utility of LDCT in our population, SATS recommends a conservative threshold for a nodule to be considered positive at baseline ( $\geq 6 \mathrm{~mm}$ ), and that pre-existing nodules should demonstrate growth (27). This is in line with the revised Lung-RADS ${ }^{\mathrm{TM}} 1.1$ guidelines, which would categorize solid and partly solid nodules $<6 \mathrm{~mm}$ as benign or to have an appearance consistent with a negative scan (25). If a non-calcified solid or partly solid nodule is $\geq 6 \mathrm{~mm}$, but $<10 \mathrm{~mm}$ with no malignant features (e.g., clearly spiculated margins), the LDCT should be repeated in 6 months. If a solid nodule or the largest solid component of a non-solid nodule is $\geq 10$ or $\geq 6 \mathrm{~mm}$ and it is enlarging or has additional malignant features, definitive action to exclude lung cancer is warranted (Figure 1). Appropriate investigations in this setting would include a standard contrasted CT scan of the chest and upper abdomen, followed by tissue sampling (either percutaneously, endoscopically, or surgically). Non-surgical tissue sampling may have an appreciable diagnostic yield for TB and could therefore avoid futile surgical intervention (39). A positron emission tomographycomputed tomography (PET/CT) scan may be used to guide further management, but will be of limited value in distinguishing between TB or malignancy, unless the standardized uptake value (SUV) value is $>5$, in which case the PET scan has a high specificity for cancer (40).

\section{Surgical intervention and potential downsides}

Lung cancer screening has the potential to save lives. The downside, however, is the potential for unnecessary surgery and the resultant morbidity that may ensue (13). This stresses the importance of a dedicated team with management decisions made by consensus. Stage I lung cancer is amenable to minimally invasive thoracic surgery (6). Video-assisted thoracic surgery (VATS) which started in the early 90 s and was initially utilized as a diagnostic procedure is now used to perform lobectomies, segmentectomies, removal of mediastinal masses and even for lymph node dissection. VATS procedures, especially for stage I lung cancer have become the modern standard, avoiding the necessity for an open thoracotomy which has far more potential for significant morbidity and mortality (41).

\section{Concomitant program recommendations}

All hospital patients whether or not they are being investigated for lung cancer should be encouraged to stop smoking and referred to a smoking cessation program if one is available, as prevention is likely to have a far greater 
impact on lung cancer mortality than does screening (4). Stakeholder education is important, as is access to a multidisciplinary team to educate, advise and potentially manage lung cancer or other incidental findings.

\section{Conclusions}

Lung cancer is the leading cause of cancer-related death in South Africa. Early trials of chest radiograph-based screening in males at high risk for lung cancer found no mortality benefit for a radiograph alone or a strategy utilizing both a radiograph and sputum cytology. Large prospective studies, including the NLST, have shown an all-cause mortality benefit when LDCT was used as a screening modality in patients that are at high risk of developing lung cancer. The SATS, based on these findings and numerous international guidelines (42), suggest that annual LDCT should be offered to patients 55-74 years who are current or former smokers (having quit within the preceding 15 years) with at least a 30 pack year history and with no history of lung cancer. Patients should be in general good health, fit for surgery and willing to undergo further investigations if deemed necessary. Given the high local prevalence of tuberculous lung disease, we recommend a conservative threshold of $\geq 6 \mathrm{~mm}$ for a positive baseline screen. If a non-calcified solid or partly solid nodule is $\geq 6 \mathrm{~mm}$, but $<10 \mathrm{~mm}$ with no malignant features (e.g., with a clearly spiculated margin), the LDCT should be repeated in 6 months. If a solid nodule or the largest solid component of a non-solid nodule is $\geq 10$ or $\geq 6 \mathrm{~mm}$ and enlarging or has additional malignant features present, definitive action to exclude lung cancer is warranted. Patients without these features should be screened annually until 15 years have elapsed from the date of smoking cessation, they turn 80 , become unfit for surgery or significant changes are observed.

\section{Acknowledgments}

We acknowledge that the full SATS council (available at https://pulmonology.co.za/council-members/) reviewed and indorsed this statement.

Funding: K Dheda is supported by the South African MRC (RFA-EMU-02-2017) and the EDCTP (TMA2015SF-1043 \& TMA-1051-TESAII).

\section{Footnote}

Conflicts of Interest: The authors have no conflicts of interest to declare.

Ethical Statement: The authors are accountable for all aspects of the work in ensuring that questions related to the accuracy or integrity of any part of the work are appropriately investigated and resolved.

\section{References}

1. Siegel RL, Miller KD, Jemal A. Cancer statistics, 2018. CA Cancer J Clin 2018;68:7-30.

2. World Health Organization. International Agency for Research on Cancer. South Africa. Int Agency Res Cancer South Africa 2018.

3. The National Institute for Communicable Diseases (NICD). Cancer in South Africa 2014 Full Report 2014. Available online: http://www.nicd.ac.za/wp-content/ uploads/2017/03/2014-NCR-tables-1.pdf (accessed 09/07/2019)

4. van Zyl-Smit RN, Allwood B, Stickells D, et al. South African tobacco smoking cessation clinical practice guideline. S Afr Med J 2013;103:869-76.

5. Malhotra J, Malvezzi M, Negri E, et al. Risk factors for lung cancer worldwide. Eur Respir J 2016;48:889-902.

6. Detterbeck F, Boffa D, Kim A, et al. The Eighth Edition Lung Cancer Stage Classification. Chest 2017;151:193-203.

7. Molina JR, Adjei AA, Jett JR. Advances in chemotherapy of non-small cell lung cancer. Chest 2006;130:1211-9.

8. Koegelenberg CF, Aubeelack K, Nanguzgambo AB, et al. Adenocarcinoma the most common cell type in patients presenting with primary lung cancer in the Western Cape. S Afr Med J 2011;101:321.

9. John TJ, Plekker D, Irusen EM, et al. Does access to private healthcare influence potential lung cancer cure rates? S Afr Med J 2017;107:687-90.

10. Patz EF Jr, Goodman PC, Bepler G. Screening for lung cancer. N Engl J Med 2000;343:1627-33.

11. van der Aalst CM, van den Bergh KA, Willemsen MC, et al. Lung cancer screening and smoking abstinence: 2 year follow-up data from the Dutch-Belgian randomised controlled lung cancer screening trial. Thorax 2010;65:600-5. 
12. Fan L, Fan K. Lung cancer screening CT-based coronary artery calcification in predicting cardiovascular events: A systematic review and meta-analysis. Medicine (Baltimore) 2018;97:e10461.

13. Croswell JM, Baker SG, Marcus PM,et al. Cumulative incidence of false-positive test results in lung cancer screening: a randomized trial. Ann Intern Med 2010;152:505-12.

14. Woolf SH, Harris RP, Campos-Outcalt D. Low-dose computed tomography screening for lung cancer: how strong is the evidence? JAMA Intern Med 2014;174:2019-22.

15. Brenner DJ. Radiation risks potentially associated with low-dose CT screening of adult smokers for lung cancer. Radiology 2004;231:440-5.

16. National Lung Screening Trial Research Team, Church TR, Black WC, et al. Results of initial low-dose computed tomographic screening for lung cancer. N Engl J Med 2013;368:1980-91.

17. van Zyl Smit RN, Pai M, Yew WW, et al. Global lung health: the colliding epidemics of tuberculosis, tobacco smoking, HIV and COPD. Eur Respir J 2010;35:27-33.

18. Berlin N, Buncher C, Fontana R, et al. The National Cancer Institute Cooperative Early Lung Cancer Detection Program. Results of the initial screen (prevalence). Early lung cancer detection: Introduction. Am Rev Respir Dis1 1984;130:545-9.

19. Oken MM, Hocking WG, Kvale PA, et al. Screening by chest radiograph and lung cancer mortality: the Prostate, Lung, Colorectal, and Ovarian (PLCO) randomized trial. JAMA 2011;306:1865-73.

20. Larke FJ, Kruger RL, Cagnon CH, et al. Estimated radiation dose associated with low-dose chest CT of average-size participants in the National Lung Screening Trial. AJR Am J Roentgenol 2011;197:1165-9.

21. Lindsay R, Newman RT, Speelman WJ. A study of airborne radon levels in Paarl houses (South Africa) and associated source terms, using electret ion chambers and gamma-ray spectrometry. Appl Radiat Isot 2008;66:1611-4.

22. Black WC. Computed tomography screening for lung cancer: review of screening principles and update on current status. Cancer 2007;110:2370-84.

23. Kazerooni EA, Austin JH, Black WC, et al. ACR-STR practice parameter for the performance and reporting of lung cancer screening thoracic computed tomography (CT): 2014 (Resolution 4). J Thorac Imaging 2014;29:310-6.

24. Optican RJ, Chiles C. Implementing lung cancer screening in the real world: opportunity, challenges and solutions. Transl Lung Cancer Res 2015;4:353-64.
25. Clark TJ, Flood TF, Maximin ST, et al. Lung CT Screening Reporting and Data System Speed and Accuracy Are Increased With the Use of a Semiautomated Computer Application. J Am Coll Radiol 2015;12:1301-6.

26. Beyer SE, McKee BJ, Regis SM, et al. Automatic LungRADS $^{\mathrm{TM}}$ classification with a natural language processing system. J Thorac Dis 2017;9:3114-22.

27. Pinsky PF, Gierada DS, Black W, et al. Performance of LungRADS in the National Lung Screening Trial: a retrospective assessment. Ann Intern Med 2015;162:485-91.

28. Fan L, Liu SY. Lung cancer screening using low dose CT: screening population and positive results definition. J Thorac Dis 2015;7:E338-40.

29. Yousaf-Khan U, van der Aalst C, de Jong $P$, et al. Final screening round of the NELSON lung cancer screening trial: the effect of a 2.5-year screening interval. Thorax 2017;72:48-56.

30. Walter JE, Heuvelmans MA, de Jong PA, et al. Occurrence and lung cancer probability of new solid nodules at incidence screening with low-dose CT: analysis of data from the randomised, controlled NELSON trial. Lancet Oncol 2016;17:907-16.

31. Horeweg N, Scholten E, de Jong P, et al. Detection of lung cancer through low-dose CT screening (NELSON): a prespecified analysis of screening test performance and interval cancers. Lancet Oncol 2014;15:1342-50.

32. Henschke CI, Yip R, Yankelevitz DF, et al. Computed tomography screening for lung cancer. Ann Intern Med 2013;159:156-7.

33. Yip R, Henschke C, Yankelevitz D, et al. CT screening for lung cancer: alternative definitions of positive test result based on the national lung screening trial and international early lung cancer action program databases. Radiology 2014;273:591-6.

34. Usman Ali M, Miller J, Peirson L, et al. Screening for lung cancer: A systematic review and meta-analysis. Prev Med 2016;89:301-14.

35. Armstrong K, Kim J, Halm E, et al. Using lessons from breast, cervical, and colorectal cancer screening to inform the development of lung cancer screening programs. Cancer 2016;122:1338-42.

36. World Health Organization. WHO Global tuberculosis report 2018. Available online: https://www.who.int/tb/ publications/global_report/en/

37. Hahm CR, Park HY, Jeon K, et al. Solitary pulmonary nodules caused by Mycobacterium tuberculosis and Mycobacterium avium complex. Lung 2010;188:25-31.

38. Chung CL, Chen YF, Lin Y'T, et al. Outcome of untreated 
lung nodules with histological but no microbiological evidence of tuberculosis. BMC Infect Dis 2018;18:530.

39. Fukuda H, Ibukuro K, Tsukiyama T, et al. CT-guided transthoracic core biopsy for pulmonary tuberculosis: diagnostic value of the histopathological findings in the specimen. Cardiovasc Intervent Radiol 2004;27:226-30.

40. du Toit R, Shaw JA, Irusen EM, et al. The diagnostic accuracy of integrated positron emission tomography/ computed tomography in the evaluation of pulmonary

Cite this article as: Koegelenberg CF, Dorfman S, Schewitz I, Richards GA, Maasdorp S, Smith C, Dheda K; on behalf of the South African Thoracic Society. Recommendations for lung cancer screening in Southern Africa. J Thorac Dis 2019;11(9):36963703. doi: $10.21037 /$ jtd.2019.08.66 mass lesions in a tuberculosis-endemic area. S Afr Med J 2015;105:1049-52.

41. Shah RD, D'Amico TA Modern impact of video assisted thoracic surgery. J Thorac Dis 2014;6:S631-6.

42. Jaklitsch MT, Jacobson FL, Austin JH, et al. The American Association for Thoracic Surgery guidelines for lung cancer screening using low-dose computed tomography scans for lung cancer survivors and other high-risk groups. J Thorac Cardiovasc Surg 2012;144:33-8. 